\title{
Kriging-assisted robust black-box simulation optimization in direct speed control of DC motor under uncertainty
}

\begin{abstract}
In spite of the wide improvement in computer simulation packages, analyzing, and optimizing the simulation model, particularly under uncertainty can still be computationally expensive and time-consuming. This paper aims to tackle these features by proposing a comprehensive methodology applied to black-box stochastic simulation models under uncertainty. For this purpose, the common surrogate model as Kriging metamodel is served to fit the simulation input-output data produced by Latin hypercube sampling experimental design. Taguchi terminology of robust design enables the optimization methods to control uncertainty and uncontrollable environmental factors. So as to formulate robust counterpart optimization, three different models in the class of dual-response surface are integrated with metamodel and robust design. Leave-one-out cross-validation is applied to validate the Kriging metamodel. Finally, a numerical case study as a direct speed control of dc motor under uncertainty is served to demonstrate the applicability of the proposed method in real engineering problems. This simplified and practical mechatronics case illustrates how the proposed procedure can be expanded for analyzing and optimizing the real complex systems.
\end{abstract}

Keyword: Uncertainty; Computational modeling; Robustness; DC motors; Optimization; Analytical models; Mathematical model 